\title{
Relationship of glycaemic level of mother in last two hours before delivery and occurrence of acute neonatal complications
}

\section{Georgy Joy Eralil*}

\begin{abstract}
Department of Obstetrics \& Gynaecology, Sreenarayana Institute of Medical Sciences, Mahatma Gandhi University, Kochi, Kerala, India
\end{abstract}

Received: 29 July 2014

Accepted: 9 August 2014

\author{
*Correspondence: \\ Dr. Georgy Joy Eralil, \\ E-mail: georgy_eralil@hotmail.com
}

(C) 2014 Eralil GJ. This is an open-access article distributed under the terms of the Creative Commons Attribution NonCommercial License, which permits unrestricted non-commercial use, distribution, and reproduction in any medium, provided the original work is properly cited.

\begin{abstract}
Background: Diabetes is the most common pre-existing medical disorder seen in pregnancy. Between 2-5\% of pregnancies are complicated by diabetes. $87.5 \%$ of these women have gestational diabetes, $7.5 \%$ have type 1 and $5 \%$ have type 2 . The study was designed to know the relation of control of diabetes to neonatal outcome in terms of metabolic complications.

Methods: Study was conducted in laboring women in government medical college hospital Kottayam. Selection criteria was based on oral GTT. Patients with multiple gestations, other medical disorders were excluded. All the patients were monitored with hourly RBS in active phase of labor. It was compared with cord levels of RBS, calcium, haematocrit and admission to SCNU. For data measured at normal level Chi square test was used to study the significance of difference between the groups. For statistically significant differences odds ratios were computed and 95\% confidence limits are calculated. Whenever necessary for quantitative data t- test was used to study the difference in mean values.

Results: All normal RBS had live births and all the intrauterine deaths were associated with maternal hyperglycaemia. Among the SCNU admission $87.5 \%$ had hyperglycaemia in mother. $33.3 \%$ of abnormal RBS had admission to SCNU whereas only $3.4 \%$ of the normal RBS group had admission. The risk of SCNU admission is 14 times more among babies of mothers with abnormal maternal RBS. $X^{2}=16.188 \mathrm{P}=0.000$. The correlation coefficient between maternal RBS and cord RBS is 0.35 indicating a negative relationship between maternal RBS and Cord RBS. The coefficient of determination $\left(\mathrm{r}^{2}\right)$ is 12 indicates $12 \%$ of variation of RBS is explained by maternal RBS. The average RBs cord among mothers with abnormal maternal RBS is 58.4 and among mothers with normal RBS is 72.4 and the observed difference is statistically significant.

Conclusions: Heart The study shows that tight regulation of maternal glucose levels during labor can reduce the incidence of neonatal hypoglycaemia and is needed for best neonatal outcome.
\end{abstract}

Keywords: GDM, Hypoglycaemia, RBS, Hypocalcaemia, Oral glucose tolerance test, Neonatal outcome

\section{INTRODUCTION}

Diabetes is the most common pre-existing medical disorder seen in pregnancy. Between $2-5 \%$ of pregnancies are complicated by diabetes. $87.5 \%$ of these women have gestational diabetes, $7.5 \%$ have type 1 and $5 \%$ have type 2 .
Gestational diabetes is defined by the World Health Organization (WHO) as 'carbohydrate intolerance resulting in hyperglycaemia of variable severity with onset or first recognition during pregnancy'.

For many years there was no consensus on the best method of screening diagnostic criteria, definition, management or treatment of gestational diabetes. 
This evidence now suggests that there is a continuum of risk with hyperglycaemia in pregnancy. It remains unclear at what level to define 'gestational diabetes', not helped by differing testing regimens and definitions in the key trials. As such there is significant variation nationally and internationally as to the cut off for diagnosis. NICE recommends a fasting glucose of $>6 \mathrm{mmol} / \mathrm{l}$ and 2-hour post prandial $>7.8$ for diagnosis.

International Association of Diabetes in Pregnancy Study Groups (IADPSG) criteria for Gestational Diabetes Mellitus (GDM) The thresholds were fasting: $92 \mathrm{mg} / \mathrm{dL}$ (5.1 mmol/L), 1 hour: $180 \mathrm{mg} / \mathrm{dL}(10.0 \mathrm{mmol} / \mathrm{L})$, and 2 hour postload: $153 \mathrm{mg} / \mathrm{dL}(8.5 \mathrm{mmol} / \mathrm{L})$.

\section{METHODS}

Study was conducted in laboring women in government medical college hospital, Kottayam. Selection criteria were based on oral GTT. Patients with multiple gestations, other medical disorders were excluded.

All the patients were monitored with hourly RBS in active phase of labor. It was compared with cord levels of RBS, calcium, haematocrit and admission to SCNU.

For data measured at normal level Chi square test was used to study the significance of difference between the groups. For statistically significant differences odds ratios were computed and $95 \%$ confidence limits are calculated. Whenever necessary for quantitative data t- test was used to study the difference in mean values.

\section{RESULTS}

All normal RBS had live births and all the intrauterine deaths were associated with maternal hyperglycaemia. Among the SCNU admission $87.5 \%$ had hyperglycaemia in mother. $33.3 \%$ of abnormal RBS had admission to SCNU whereas only $3.4 \%$ of the normal RBS group had admission. The risk of SCNU admission is 14 times more among babies of mothers with abnormal maternal RBS. $\mathrm{X}^{2}=16.188 \mathrm{P}=0.000$.

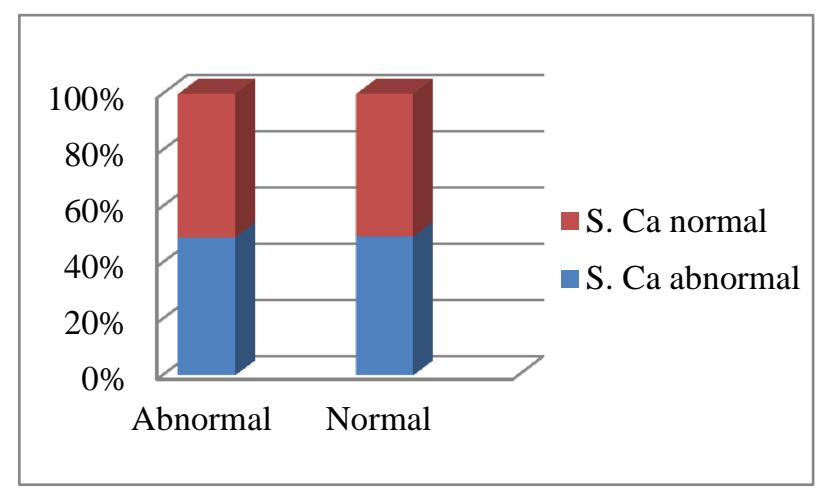

Figure 1: Distribution of serum calcium of babies among abnormal and normal maternal RBS.
Among mothers with abnormal RBS 57.1\% had neonatal hypocalcaemia. The corresponding percentage is only $41.4 \%$ among mothers with normal maternal RBS. $X^{2}=$ $2.425 \mathrm{P}=0.119$, the observed difference is not statistically significant. $14.3 \%$ of hyperglycaemic mothers had neonatal hyperbilirubinaemia. All the neonatal hyperbilirubinemia was associated with hyperglycaemia in mother. There is significant relationship between maternal RBS and neonatal hyperbilirubinaemia. $\mathrm{X}^{2}=8.815 \mathrm{P}=0.003$.

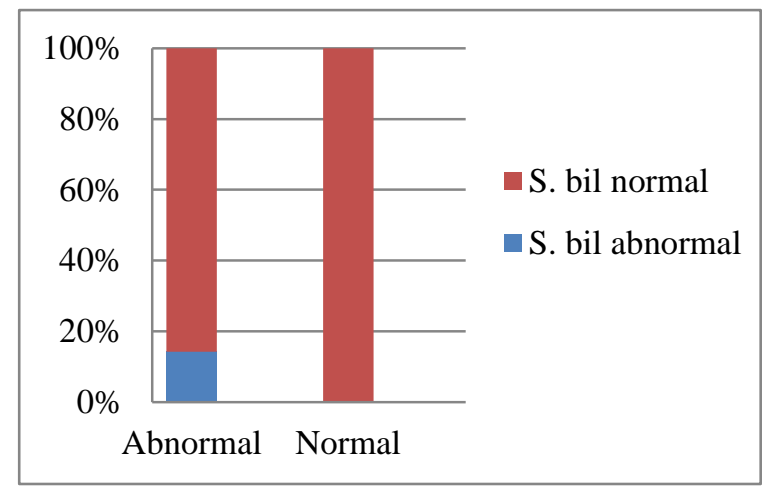

Figure 2: Distribution of serum bilirubin of babies among abnormal and normal maternal RBS.

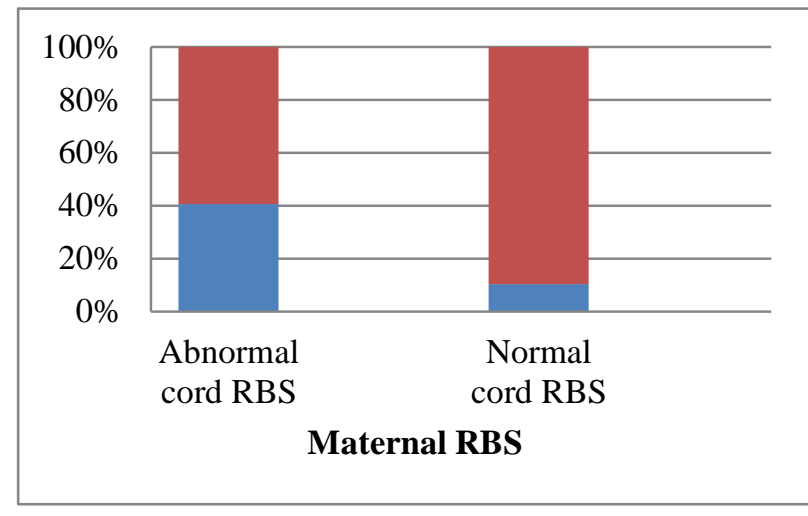

Figure 3: Distribution of RBS cord among abnormal and normal maternal RBS.

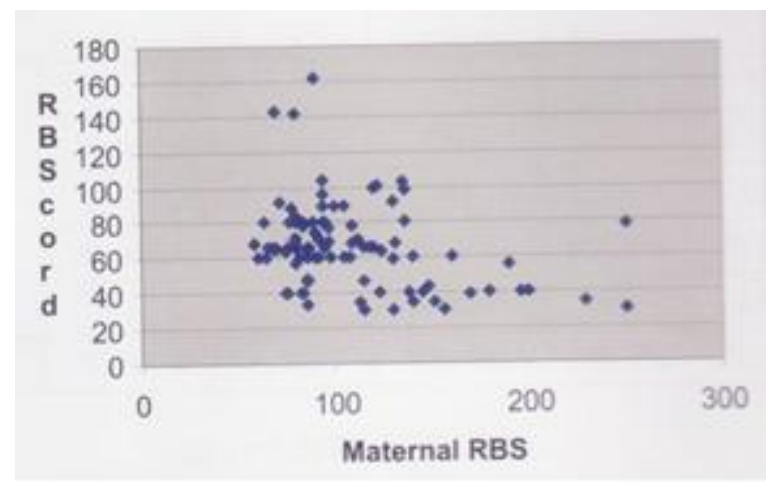

Figure 4: Correlation between maternal RBS and RBS cord. 
The odds ratio is 5.8 and $95 \%$ confidence limits for the odds ratio are 2 and 16.7.the risk of having abnormal cord RBS is 5.8 times among mothers with abnormal maternal RBS. $\mathrm{X}^{2}=12.488 \mathrm{P}=0.000$.

The correlation coefficient between maternal RBS and cord RBS is 0.35 indicating a negative relationship between maternal RBS and cord RBS. The coefficient of determination $\left(\mathrm{r}^{2}\right)$ is 12 indicates $12 \%$ of variation of RBS is explained by maternal RBS.

The average RBS cord among mothers with abnormal maternal RBS is 58.4 and among mothers with normal RBS is 72.4 and the observed difference is statistically significant (Table 1).

Table 1: Average RBS cord among mothers with abnormal maternal RBS.

\begin{tabular}{|c|c|c|c|}
\hline & Mean \pm SD & $\mathbf{T}$ & Significance \\
\hline Abnormal & $58.4 \pm 22.93901$ & \multirow{2}{*}{2.96} & \multirow{2}{*}{0.003} \\
\hline Normal & $72.4 \pm 23.49893$ & & \\
\hline
\end{tabular}

\section{DISCUSSION}

Nearly third of mothers belonged to 25-29 years of age. The groups were comparable with respect to age.

All euglycaemic mothers had live births and all intrauterine deaths were associated with maternal hyperglycaemia.

Among the NICU admission $87.5 \%$ had hyperglycaemia in mother $33.3 \%$ of abnormal RBS had admission to SCNU whereas only $3.4 \%$ of normal RBS group had admission. Risk of SCNU admission is 14 times more among babies of mothers with abnormal maternal RBS.

Among mothers with abnormal RBS $57.1 \%$ had neonatal hypocalcaemia. $14.3 \%$ of hyperglycaemic mothers had neonatal hyperbilirubinaemia.

The odds ratio is 5.8 and $95 \%$ confidence limits for odds ratio are 2 and 16.7. The risk of having abnormal cord RBS is 5.8 times among mothers with abnormal maternal RBS. The correlation coefficient between maternal RBS and cord RBS is -0.35 indicating a negative relationship between maternal RBS and RBS cord. The coefficient of determination is 12 indicating $12 \%$ of variation is explained by maternal RBS.

Lowering glucose is of pivotal importance in the treatment of diabetes in pregnancy. ${ }^{1}$ A spectrum of different glucose thresholds can be established and used appropriately to prevent each complication. This article outlines the concept of normality and what definition of normality should be used to evaluate the relationship between the level of glycaemia and perinatal outcome.

In GDM, it has been established that there is a positive relationship between levels of maternal glycaemia and perinatal morbidity and mortality. ${ }^{2}$ Although the terms normal and abnormal seem to be self-explanatory, the range suggested for the measurement of a specific medical condition is often incompatible for the stated goals. As a result, there is a need to establish thresholds or boundaries for targeted glycaemic levels appropriate to a specific diabetic complication. ${ }^{4}$

Perinatal mortality in pre-existing diabetes was higher than in the GDM patients but with similar rates between type 1 (stillbirth: 12/1000; neonatal death: 8/1000) and type 2 diabetes (stillbirth: 13/1000; neonatal death: $5 / 1000) .{ }^{5}$ The higher rate of perinatal mortality in the preexisting diabetic patients is attributable in part to the lower rate of those achieving targeted levels of glycaemic control and to the higher incidence of congenital malformations and vascular complications. ${ }^{6}$

The abnormal maternal glucose level causes cellular hyperplasia and hypertrophy of most foetal tissues resulting in foetal hyperinsulinaemia. The consequence of this abnormality is macrosomia, metabolic and respiratory complications, and others. As the common denominator is maternal hyperglycaemia and foetal hyperinsulinaemia, it is reasonable to speculate that glycaemic control on the maternal side will positively influence the foetal side. ${ }^{7}$

\section{ACKNOWLEDGEMENTS}

Author would like to thank Prof. Mariam Thomas, head of department of obstetrics and gynaecology and prof. Kunjamma Roy, department of obstetrics and gynaecology for their support.

Funding: No funding sources

Conflict of interest: None declared

Ethical approval: The study was approved by the institutional ethics committee

\section{REFERENCES}

1. V. Rosenberg, G. Eglinton, E. Rauch, D. Skupski. Intrapartum maternal glycemic control in women with insulin requiring diabetes a randomized clinical trial of rotating fluids versus insulin drip. Am J Obstet Gynaecol. 2006;195:1095-9.

2. A. Jamal, N. Choobak, F. Tabassomi. Intrapartum maternal glucose infusion and fetal acid base status. Int J Gynaecol Obstet. 2007;97:187-9.

3. Rosenberg, Victor A, Eglinton, Gary S, Eden R, Skupski, et al. Intrapartum maternal glycaemic control in women with insulin requiring diabetes: a randomized clinical trial of rotating fluids versus insulin drip. Obstet Gynaecol Surv. 2007 Mar;62(3):159-61.

4. J. Mendiola, L. J. Grylack, J. W. Scanlon. Effects of intrapartum maternal glucose infusion on the normal foetus and newborn. Anaesth Analg. 1982 Jan;61(1):32-5. 
5. Jovanovic L, Peterson CM. Insulin and glucose requirements during first stage of labor in insulin dependent diabetic women. Am J Med. 1983;75:607.

6. Gajjar F, Maitra NK. Intrapartum and perinatal outcomes in women with gestational diabetes and mild gestational hyperglycaemia. J Obstet Gynaecol India. 2005;55:135-7.
7. Curet LB, Izquierdo LA, Gilson GJ, Schneider JM, Perelman R, Converse J. Relative effects of antepartum and intrapartum maternal blood glucose levels on the incidence of neonatal hypoglycaemia. $\mathbf{J}$ Perinatol. 1997 Mar-Apr;17(2):113-5.

DOI: $10.5455 / 2320-1770 . i j r \operatorname{cog} 20140966$

Cite this article as: Eralil GJ. Relationship of glycaemic level of mother in last two hours before delivery and occurrence of acute neonatal complications. Int J Reprod Contracept Obstet Gynecol 2014;3:692-5. 